\title{
RIED. Una historia de 30 años de publicación científica
}

\section{RIED. A 30-year history of scientific publication}

\author{
Lorenzo García Aretio \\ UNED (España)
}

\section{Resumen}

La “Asociación Iberoamericana de Educación Superior a Distancia” (AIESAD) es, probablemente, la más relevante de las organizaciones centradas en la educación abierta y a distancia en Iberoamérica y fundada en 1980. Uno de los objetivos fundamentales que se propuso lograr esta asociación fue el de crear una publicación académica periódica como medio ideal para la divulgación científica y mejora de los procesos educativos no presenciales. Así, en 1982 se crea el "Boletín Informativo de la Asociación Iberoamericana de Educación Superior a Distancia”. Este boletín puso las bases para el nacimiento real de una revista científica, la "Revista Iberoamericana de Educación Superior a Distancia" (RIESAD) cuyo primer número fue publicado en 1988 y el último en 1996, con un total de 172 artículos. En 1998 esta publicación se actualizó bajo un nombre similar, "RIED. Revista Iberoamericana de Educación a Distancia" que, en la actualidad, adaptándola a los tiempos, lleva el subtítulo de "Revista Iberoamericana de la Educación Digital” y que hasta este número actual ha publicado un total de 513 artículos. Por tanto, 30 años de historia (1988-2018) de una publicación científica, con un total de 685 trabajos que vieron la luz sobre educación a distancia y tecnologías digitales aplicadas a la enseñanza y al aprendizaje.

Palabras clave: AIESAD; RIESAD; RIED; educación a distancia; educación digital; revista; iberoamericana; historia.

\begin{abstract}
Founded in 1980, the IberoAmerican Association for Distance Higher Education ("Asociación Iberoamericana de Educación Superior a Distancia” or AIESAD) is probably the most relevant organization of open and distance education in IberoAmerica. One of the fundamental objectives of this association was to create a periodic academic publication as an ideal medium for the scientific dissemination and improvement of non-face-toface educational processes. Thus, in 1982 the "Informative Bulletin of the IberoAmerican Association of Distance Higher Education" was created. This bulletin laid the foundations for the real inception of a scientific journal, the "IberoAmerican Journal of Distance Higher Education" (Revista Iberoamericana de Educación Superior a Distancia or RIESAD) whose first issue was published in 1988 and the last one in 1996, with a total of 172 articles. In 1998 this publication was updated under a similar name, "IberoAmerican Journal of Distance Education” (RIED. Revista Iberoamericana de Educación a Distancia). As a way of adapting
\end{abstract}


to modern tendencies, it bears the subtitle of "Iberoamerican Journal of Digital Education" and, up to this current issue, it has published a total of 513 articles. These are the 30 years of history (1988-2018) of a scientific publication, with a total of 685 papers that came to light on distance education and digital technologies applied to teaching and learning.

Keywords: AIESAD, RIESAD, RIED, distance education, digital education, magazine, IberoAmerican, history.

La Asociación Iberoamericana de Educación Superior a Distancia (AIESAD) es, probablemente, la más relevante de las organizaciones en educación abierta y a distancia en Iberoamérica. La AIESAD fue fundada en octubre de 1980 en Madrid, durante el I Simposio Iberoamericano de Rectores de Universidades Abiertas en el que participaron la Universidad Nacional de Educación a Distancia-UNED(España), Universidad Estatal a Distancia-UNED- (Costa Rica), Universidad Nacional Abierta -UNA- (Venezuela), Universidad de la Sabana -UNISABANA(Colombia), Universidad de Brasilia -UnB- (Brasil) y la Universidad Nacional Autónoma-UNAM- (México).

Componen la AIESAD universidades abiertas y a distancia, así como consorcios de educación a distancia y universidades mixtas (con modalidad presencial y a distancia). La idea fundacional era la de avanzar en pro de una mejor educación y aprendizaje a distancia y de promover el intercambio entre las universidades a distancia en Iberoamérica. Sus fundadores consideraron necesario crear un instrumento permanente para facilitar el flujo de información en el área de educación superior a distancia, ya fuese a través de mecanismos de cooperación universitaria, de una mayor coordinación institucional a través de proyectos conjuntos, o de la puesta en común de sus buenas prácticas en la materia.

Debe reconocerse que la Asociación Iberoamericana de Educación Superior a Distancia (AIESAD) ha sido pionera en la implantación y difusión de la educación a distancia (EaD) en el nivel de la educación superior. En un tiempo en el que no se reconocía la calidad de esta metodología, supo aunar esfuerzos e impulsar iniciativas educativas capaces de llegar a todos los sectores poblacionales. Ahora bien, no debemos obviar las diferentes experiencias educativas apoyadas en la $\mathrm{EaD}$ que se estaban dando antes de su creación en toda América Latina, germen de la actual difusión y reconocimiento de esta metodología. México, Colombia, Venezuela, Brasil o Ecuador, por ejemplo, presentan una historia muy sugerente de estas experiencias, tanto en el ámbito de la educación formal como no formal. Pero lo que aportó la AIESAD a todas estas iniciativas fue la propuesta de una asociación que uniera estos esfuerzos, cooperara en su mantenimiento y aumentara su visibilidad entre todos estos países. En un tiempo en el que aún no se valoraba el potencial de las redes de colaboración, la AIESAD fue capaz de adelantarse y proponer un instrumento de 
cooperación. Y, más aún, apostó por la educación superior como nivel clave para el desarrollo de esta región.

De acuerdo con los fines y objetivos que se propuso lograr, resultó lógico crear una publicación académica periódica como medio ideal para plasmar y difundir esos fines, a la vez que como canal eficaz y eficiente para su logro. Precisamente, en un estudio detenido sobre la relevancia del esfuerzo de la AIESAD por el ámbito de las publicaciones científicas, García Aretio y Ruíz Corbella (2011) concluyeron lo siguiente:

- La constatación de la relevancia de las publicaciones periódicas científicas para recoger el progreso de un ámbito del saber.

- La toma de conciencia a nivel mundial y, específicamente, iberoamericano en cuanto a la relevancia de las revistas científicas para la promoción del conocimiento.

- La importancia de un campo como el de la educación a distancia, emergente en el último tercio del siglo pasado y muy consolidado e innovador en el momento actual.

- La relevancia de la Asociación Iberoamericana de Educación Superior a Distancia (AIESAD) como organización pionera en la divulgación de la educación a distancia en toda su área de influencia, con el inicio en 1982 de su primera publicación periódica, el Boletín Informativo de la Asociación Iberoamericana de Educación Superior a Distancia.

- La evolución y adecuación progresiva a los diferentes criterios de calidad de las tres etapas de publicaciones periódicas de la AIESAD: a) Boletín Iberoamericano de la Asociación Iberoamericana de Educación Superior a Distancia, 19821988, b) Revista Iberoamericana de Educación Superior a Distancia (RIESAD), 1988-1996, c) RIED. Revista Iberoamericana de Educación a Distancia, 1998-actualidad.

- La constatación final de que la AIESAD y sus publicaciones científicas suponen una contribución que merecería del reconocimiento internacional por este esfuerzo de divulgación del conocimiento científico en el ámbito de la educación a distancia. 


\section{EL BOLETÍN INFORMATIVO DE LA ASOCIACIÓN IBEROAMERICANA DE EDUCACIÓN SUPERIOR A DISTANCIA}

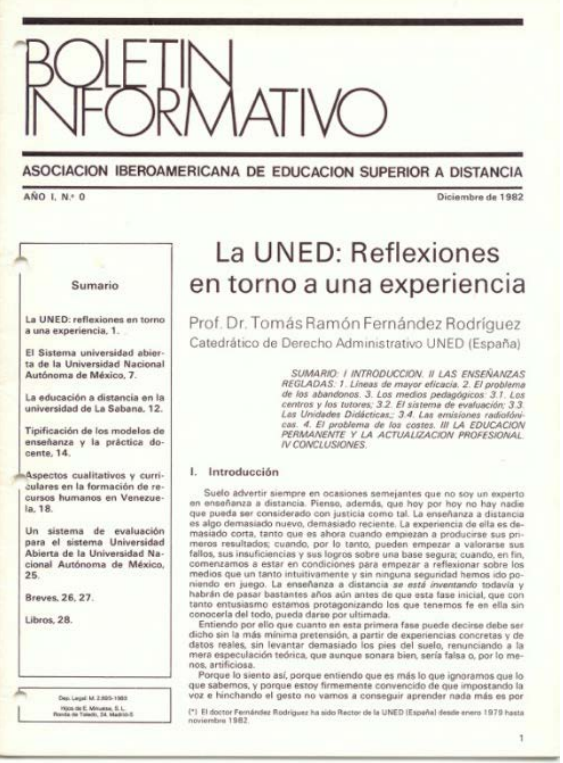

Con esa intención de crear una publicación periódica, un año después de nacer, en 1982, la AIESAD lanza el Boletín Informativo de la Asociación Iberoamericana de Educación Superior a Distancia, como primer órgano de comunicación entre todos los socios. Estos boletines en su contenido, fundamentalmente, relataban la experiencia de diferentes países en la aplicación de la EaD, así como la comunicación de noticias y reseñas de las asambleas y Encuentros Iberoamericanos de Educación Superior a Distancia (Miranda y Yee, 2014).

Como relata Varela (2011), la publicación de este boletín arranca en diciembre de 1982 y se mantiene, con cinco ediciones anuales en los meses de febrero, abril, junio, octubre y diciembre, y así sucedió hasta febrero de 1988. A lo largo de este período, se publican un total de 24 números, dos de ellos extraordinarios. Se trata de una publicación de extensión variable, aunque generalmente reducida, que en ningún caso llegó a superar las 40 páginas. Tanto por su maquetación como por su sencilla encuadernación, grapada en las páginas centrales, este boletín recalcaba su carácter informativo al editarse en un formato más similar al de una publicación destinada a la comunicación e información de noticias destacadas entre sus asociados, así como a la divulgación de temas educativos de interés. Se trata, no obstante, de la primera publicación en español, especializada en $\mathrm{EaD}$, que presenta información del panorama internacional, fundamentalmente de Iberoamérica.

$\mathrm{Al}$ analizar atentamente sus contenidos se comprueba que, ya desde los primeros números, esta publicación se convierte en un excelente canal comunicativo mediante el que expertos e instituciones latinoamericanas dedicadas a la educación a distancia difunden conocimientos y dan a conocer sus experiencias en este ámbito. Estas aportaciones de gran calidad compartirán también espacio con otras informaciones, como convocatorias o crónicas de eventos, reglamentos, actas, proyectos, publicaciones recibidas en la sede de la AIESAD, etc.

Teniendo en cuenta la naturaleza de esta primera publicación de la AIESAD, obviamente la educación a distancia desde sus diferentes vertientes, dimensiones y formulaciones sería tema preferente de sus contenidos. Así, se vinieron tratando sobre esta modalidad: aspectos conceptuales, características, perfiles de estudiantes, 
variables curriculares, funciones y necesidad de la EaD, logros, expectativas, debilidades, prospectiva, las teorías del aprendizaje en las que se fundamenta, etc.

El Consejo Editorial de este boletín estuvo inicialmente presidido por Elisa Pérez Vera, entonces rectora de la UNED y presidenta de la AIESAD (primera mujer rectora de una universidad en España). Esa presidencia del Consejo Editorial fue desempeñada por Elisa Pérez, precisamente hasta el penúltimo número del boletín. En el último número, publicado en febrero de 1988, aparece ya como presidente de dicho consejo, Mariano Artés, rector de la UNED y presidente de la AIESAD desde 1987.

\section{LA REVISTA IBEROAMERICANA DE EDUCACIÓN SUPERIOR A DISTANCIA}

Ya con Mariano Artés como presidente de la AIESAD, en 1988 se da un salto cualitativo en lo que respecta al ámbito de las publicaciones de la AIESAD. De ahí que, dada la buena acogida del boletín, el Consejo Directivo de la asociación acuerda convertirlo en revista científica, bajo el título de Revista Iberoamericana de Educación Superior a Distancia (RIESAD) que se publicó hasta el último trimestre de 1996 (Miranda y Yee, 2014).

Esta iniciativa, ciertamente, surge a raíz del Boletín, sin ser una continuidad del mismo, señala García Pérez (2011), que añade que la RIESAD se estructura como revista científica que ofrecerá relevantes trabajos de investigación, así como experiencias sobre EaD presentadas por investigadores, generalmente procedentes de las diferentes instituciones que conforman la asociación. De ahí que cifremos en ese año 1988 el real nacimiento de RIED (de su predecesora, la RIESAD) y por eso en 2018 venimos celebrando su 30 aniversario.

En efecto, la AIESAD hace ahora 30 años, apostó en esta nueva etapa por una publicación científica impresa y periódica. El salto cualitativo que se establece desde el Boletín a la

REVISTA IBEROAMERICANA DE EDUCACION SUPERIOR A DISTANCIA

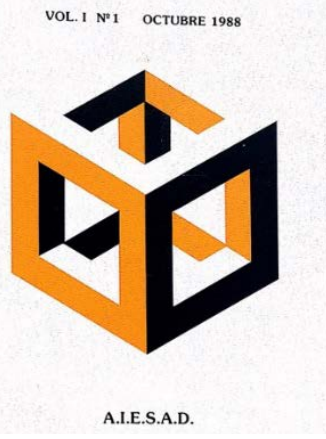
RIESAD sirvió como elemento difusor no solo orientado hacia los propios miembros de la Asociación, sino como puente de divulgación científica para docentes, investigadores y otros lectores que pudieran estar interesados en este campo emergente que auguraba un futuro prometedor. Laimportancia y el reconocimiento internacional alcanzado por esta revista de la AIESAD en los años de su publicación, residió en ser una de las escasísimas publicaciones especializadas en este ámbito tan específico, la educación a distancia. Además, es necesario 
resaltar que gracias a esta publicación se constituyó un hito de consolidación de la presencia de la lengua española en publicaciones científicas referidas a esta modalidad emergente.

Desde sus orígenes, la RIESAD contó con el apoyo, no sólo de la AIESAD, matriz de la misma, sino que además fue respaldada por dos organizaciones internacionales claves en el entorno de la EaD: el International Council of Distance Education (ICDE) y la European Association of Distance Teaching Universities (EADTU). La RIESAD surgió en formato impreso de $18 \times 24 \mathrm{~cm}$, con una portada en blanco con el logo de la AIESAD y un número medio de 125 páginas por número. El primer número se editó en 1988 y tras 8 volúmenes, y con un carácter generalmente bimensual, su último número corresponde a diciembre de 1996. Desde sus inicios contó con un Comité Científico avalado por expertos de diferentes países y un Consejo de Redacción. El primer director de la RIESAD, en 1988, vol. 1(1) fue José Luis García Garrido director, a su vez, de la Secretaría Permanente de la AIESAD y vicerrector en la UNED de España, que desempeñó el cargo hasta 1991, vol. 4(1) en que comenzó la dirección de Luis Tejero (también entonces, vicerrector de esta universidad) que la continuó hasta el último número de esta revista correspondiente al vol. 8(2), de 1996. En 1995 la presidencia de AIESAD (y el rectorado de la UNED) había pasado de Mariano Artés a Jenaro Costas. Así, fue en el mandato de este último presidente cuando esta revista dejó de publicarse. Durante todo el período de publicación de la misma, habría que destacar a una persona que, con su permanente dedicación, hizo realidad esta revista durante estos nueve años, la secretaria técnica de la misma, Pilar Velasco Mora. Fueron un total de 172 artículos los publicados en la RIESAD durante los años de su existencia.

Destacados autores encontraron en esta revista un extraordinario medio para la publicación de sus trabajos científicos. Aunque existieron estudiosos de otras áreas geográficas, la mayoría de ellos procedían de instituciones asentadas en diferentes países de Iberoamérica. Ello supuso un gran valor para la educación a distancia en este contexto geográfico en el que se estaban ofreciendo experiencias muy valiosas encaminadas a la implantación, en muchos casos y, en otros, a la consolidación de esta metodología (García Pérez, 2011). 


\section{LA RIED. REVISTA IBEROAMERICANA DE EDUCACIÓN A DISTANCIA}

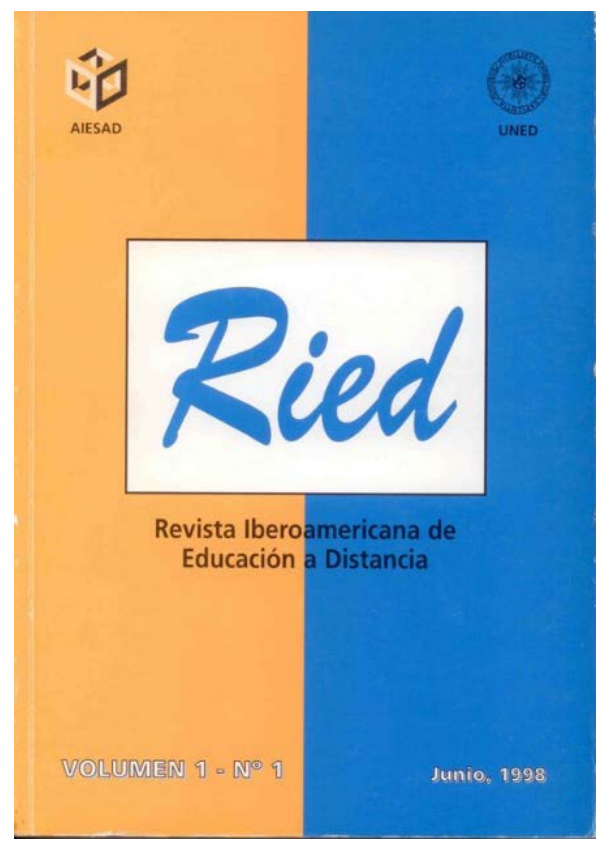

Como se ha señalado, el último número de la RIESAD vio la luz en diciembre de 1996. En ese momento se produjo un vacío en el ámbito de la publicación científica de la AIESAD, entonces presidida por Jenaro Costas. A instancias de Ramón Pérez Juste, vicerrector de la UNED, de quien dependía el Instituto Universitario de Educación a Distancia (IUED), se solicitó a quien dirigía ese instituto en aquellos años y que ahora firma este artículo, el diseño de un nuevo proyecto para la publicación de una revista científica que aprovechase el impacto de la predecesora RIESAD, fuese continuadora de la misma y que, progresivamente, asumiese todos los parámetros propios de una publicación científica de estas características, los exigidos por bases de datos $y$ agencias internacionales de evaluación de revistas científicas. Pues bien, ese proyecto fue presentado y aprobado en 1997 por el Consejo Directivo de la AIESAD. Se acordó denominar a esta revista, sucesora de la RIESAD, RIED. Revista Iberoamericana de Educación a Distancia. Se optó por eliminar el término "Superior" de la anterior denominación, con el fin de no limitar la publicación de trabajos que, sin referirse a la educación superior, podrían tener repercusión futura en ese nivel educativo (de Rivas, 2011). Así, el mandato estaba claro, convertir a la RIED, heredera de la RIESAD, en referente académico y científico de las publicaciones referidas a la modalidad no presencial, en las lenguas española y portuguesa (Miranda y Yee, 2014). Nace así la RIED, en su denominación y formato definitivos.

Debe apuntarse que, como es lógico, el entonces presidente de la AIESAD, Jenaro Costas, el director de la Secretaría Permanente de la AIESAD en aquella época, Carlos Rodrigo y el vicerrector Ramón Pérez Juste, pusieron a disposición de quien firma este trabajo, fundador y primer director de la RIED actual, los recursos necesarios para cumplir el mandato de la asociación. Para ello, nos rodeamos de dos grandes colaboradores, los profesores José María Luzón y Juan Ardoy, que actuaron como secretarios de redacción en aquellos años históricos para RIED, constituyéndose en auténticos pilares de esta nueva RIED. Con ellos y las secretarias administrativas Pilar Gabriel y Mariví Zúmel, nos dispusimos a cumplir el mandato de la AIESAD y 
en octubre de 1997 publicamos el que denominamos $\mathrm{N}^{\circ}$ o de la RIED. Se trataba de un pequeño ejemplar, ya con el formato impreso y portada de la futura revista, en el que se presentaba la publicación, sus órganos directivos y editoriales, así como la normativa propia de la misma, directrices para autores, etc.

Con estas premisas, la RIED, tal como la conocemos ahora, empezó a publicarse en 1998, con los dos números correspondientes al primer volumen, vol. 1(1 y 2). En 1999 se publicaron los dos números correspondientes a vol. 2(1 y 2). Así, con esos cuatro números, se pusieron ya las bases de lo que hoy es la RIED.

Circunstancias de la política universitaria llevaron a remover la dirección de la revista, siendo presidente de la AIESAD Jaime Montalvo. Así, a partir del año 2000 y del vol. 3(1), se sucedieron otros directores en la revista. Hasta junio de 2002, vol. 5(1) la dirección estuvo a cargo de Juan Manuel Moreno, y los números 5(2) y 6(1), a cargo de Joaquín Summers, ambos en calidad de directores de la Secretaría Permanente de la AIESAD y vicerrectores de la UNED. Desde aquel vol. 3(1) de 2000 hasta el vol. 6(1) de 2003, la editora de la revista fue Teresa Bardisa, directora del IUED de la UNED y el secretario de la revista Juan Ramón Bautista. Desde el vol. 4(2) la revista no vino cumpliendo las fechas de publicación establecidas. Los retrasos fueron evidentes y se publicaban, además, pocos artículos en cada número. Aquel vol. 6(1) en 2003 supuso la suspensión de la publicación de la RIED. En principio, en el entorno de la AIESAD y UNED, la revista se dio por muerta. La triste suspensión de la publicación se debió a diferentes causas y motivos que no sería propio analizar en este trabajo.

El Consejo Directivo de la AIESAD del año 2005 reflexionó sobre el grave problema de haber perdido su buque insignia, una publicación científica de carácter periódico, y realiza entonces el encargo de reflotar y relanzar a la RIED, a quien la fundó en 1988, en este segundo formato, y fue su primer director, el autor de este artículo, que aceptó con la condición de que la maquetación, impresión, publicación, intercambios y distribución de la revista se llevasen a cabo en la Universidad Técnica Particular de Loja (UTPL) de Ecuador, regida entonces por Luis Miguel Romero, gran impulsor de la revista, realizando tareas de directora adjunta María José Rubio, de la misma institución ecuatoriana, y otra directora adjunta en Madrid, Marta Ruíz Corbella de la UNED. Era entonces presidenta de la AIESAD Araceli Maciá, rectora de la UNED de España.

El primer objetivo, tras la suspensión de la publicación de la RIED en junio de 2003, era el de retomar los números perdidos y ajustar plenamente frecuencia y periodicidad de la revista (de Rivas, 2011). Así, desde la fecha de esta decisión de la AIESAD de reflotar a la revista, acometimos en tiempo record, a lo largo de los años 2005 y 2006, la publicación de los volúmenes atrasados 6(2) de 2003, 7(1 y 2) de 2004, 8(1 y 2) de 2005 y 9(1 y 2) de 2006. A partir del vol. 10(1), correspondiente a 2007, logramos nivelar y ajustar la publicación de la revista que, desde entonces, se ha mantenido fiel, sin un solo fallo, a la periodicidad establecida de sus dos números 
anuales, hasta este volumen actual, 21(2), correspondiente al segundo número de 2018.

Hasta el año en que dejó de publicarse, 2003, la RIED sólo se ofrecía en formato impreso. En 2006 se creó su página web en la que se volcaron en formato digital todos los números impresos ya publicados $\mathrm{y}$, a partir de ese año, la publicación vino realizándose en doble formato, impreso y digital. Desde 2006 hasta 2012, el sitio web oficial de la revista se ubicó en la Universidad Técnica Particular de Loja (UTPL) de Ecuador, institución que desde ese año 2006 viene realizando las tareas de maquetación, impresión, publicación, intercambio y distribución del formato impreso de la revista.

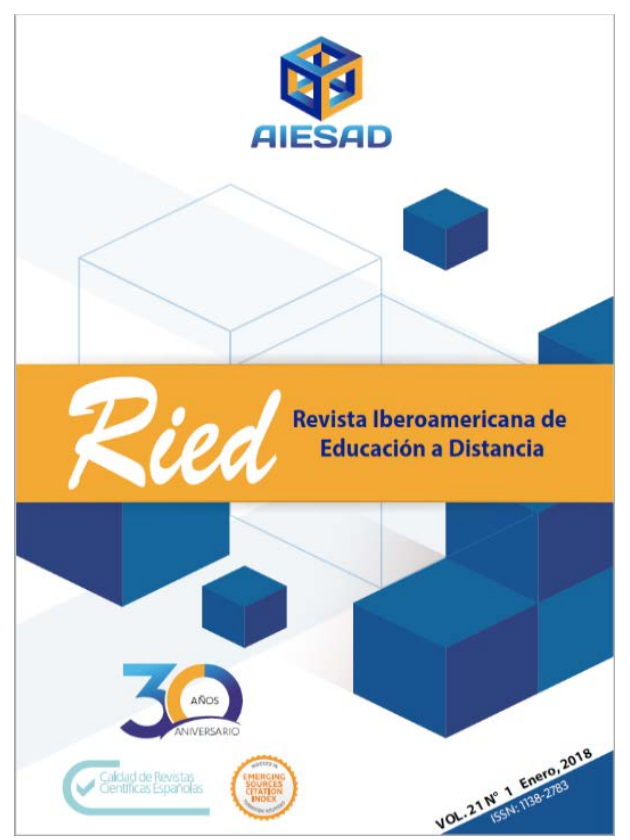

En 2012 se comienzan a migrar todos los archivos e información de la revista a la plataforma OJS (Open Journal Systems) ubicada en la UNED de España, en el área de revistas científicas de esta Universidad, con el fin de darle mayor visibilidad, más penetración y una alta fiabilidad, dado el prestigio internacional del OJS. Desde ese año 2012, la gestión íntegra de la revista se lleva a cabo desde esta plataforma. Hasta el pleno funcionamiento del OJS fueron personas clave en la gestión de la revista Marta Ruíz Corbella, María José Rubio, María García Pérez, Ángel Barbas y Beatriz Tasende. Cabría destacar a María García Pérez que como secretaria técnica de la RIED vino desempeñando una eficaz tarea durante varios años. Era entonces presidente de la AIESAD, Juan Gimeno, rector de la UNED. A quien sucedió en 2013 Carlos Eduardo Bielschowsky, rector

del CEDERJ de Brasil, quién trató insistentemente en buscar recursos que pudieran potenciar la publicación.

La gran expansión de la revista y su impacto creciente en estos últimos años, fundamentalmente desde 2014, se ha debido especialmente a las directoras adjuntas Rosario de Rivas que desde UTPL coordina con acierto todas las acciones necesarias que tiene encomendada esta universidad ecuatoriana en la publicación de la RIED, y Elena Bárcena, gran puntal de nuestra publicación, que viene coordinando al equipo de correctores de estilo que han aportado a la revista un indudable plus de calidad. Igualmente suman un gran valor las dos asistentes de dirección, Carla Netto y Carolina Schmitt que, además, realizan una encomiable labor en la difusión de RIED a través de las redes sociales. Las dos directoras adjuntas y las dos asistentes 
de dirección continúan hoy junto al mismo director/editor, en el empeño de incrementar en cada número, la calidad de esta publicación científica, a quienes se ha sumado recientemente desde la UNED, José Manuel Sáez. Una mención especial merece Iliana Ramírez, docente de la Universidad Técnica Particular de Loja (UTPL (Ecuador) y también asistente de dirección de la RIED, que desde 2005 hasta la actualidad viene desempeñando importantes tareas en la revisión de textos, maquetación, publicación impresa, distribución e intercambios de la revista. La UTPL de Ecuador ha supuesto desde 2005 un apoyo incontestable en lo que fue la recuperación de la revista, su relanzamiento y modernización, siguiendo en el empeño que inició el rector Luis Miguel Romero, quien le sucedió en el cargo de esa institución ecuatoriana, José Barbosa.

Precisamente en esta última etapa de la RIED, en 2017, se acordó subtitular la denominación de la revista. Es decir, a su nombre original y oficial de RIED. Revista Iberoamericana de Educación a Distancia, manteniéndolo, la hemos subtitulado como la Revista Iberoamericana de la Educación Digital, pudiendo conservar así las siglas de RIED y aportando una visión más moderna de la publicación, dado que sus contenidos actuales giran en torno a las tecnologías como base de propuestas educativas que propician una enseñanza y un aprendizaje digitales. Desde 2016 preside la AIESAD Luis Guillermo Carpio, rector de la UNED de Costa Rica, apoyado por el vicerrector Edgar Castro, quienes mantienen el mismo propósito de buscar recursos que faciliten el progreso de RIED.

Cabe destacar el papel fundamental de la UNED de España en este esfuerzo de AIESAD por convertirse en difusora internacional del conocimiento científico en educación a distancia y tecnologías aplicadas a la educación. Todos los rectores de esta institución española, con mayor o menor acierto, reconocieron e impulsaron el valor de una publicación científica de esta magnitud, desde Elisa Pérez Vera con quien nació el Boletín Informativo en 1982, hasta la época actual con el rector Alejandro Tiana con quien venimos a cumplir este 30 aniversario de RIED.

Sin embargo, bien es cierto que, desde el nacimiento de la RIESAD en 1988 y de la RIED (su sucesora) en 1998, nada hubiese sido posible sin los principales protagonistas, los autores que nos honraron con sus trabajos. Pero la revista se ha potenciado, sobre todo últimamente, por la calidad de los artículos en ella publicados, seleccionados entre el gran número de originales que progresivamente nos están llegando, gracias al riguroso trabajo del gran elenco de miembros del comité científico y de los evaluadores internacionales externos que realizan una excelente labor. Los lectores, estudiosos e investigadores que acuden a nuestra publicación como fuente de consulta, sea para la mejora de su práctica docente, sea para la investigación, cierran el círculo de calidad de la revista. 


\section{CONCLUSIÓN}

En suma, la AIESAD desde 1981 evolucionó con tres formulaciones resultado de una misma iniciativa: unir esfuerzos y visibilizar la ingente tarea educativa que se estaba llevando a cabo en diferentes instituciones apoyadas siempre en la metodología y recursos específicos de modalidades no presenciales. Prueba de este dinamismo es la continuidad de estas publicaciones a lo largo del tiempo, y la cantidad significativa de artículos publicados en cada una de ellas: 85 artículos en el Boletín, 174 en RIESAD y 440 en RIED (tabla 1). El análisis de estas aportaciones científicas proporciona una perfecta radiografía de la evolución de la modalidad a distancia. Cómo fue implantándose, en qué campos fueron pioneros, cuáles fueron las experiencias más significativas, cómo fueron emergiendo los diferentes escenarios, los temas recurrentes a lo largo de todos estos años, los aciertos, las fortalezas, las debilidades, las amenazas, las oportunidades y, también, los fracasos (García Aretio y Ruiz Corbella, 2011).

Tabla 1. Las publicaciones periódicas de la AIESAD (1981-2018)

\begin{tabular}{|l|c|c|c|}
\hline \multicolumn{1}{|c|}{ Revista } & Años & Vol. y núm. & $\begin{array}{c}\mathbf{N}^{\mathbf{0}} \text { de artículos } \\
\text { publicados }\end{array}$ \\
\hline $\begin{array}{l}\text { Boletín Iberoamericano de Educación } \\
\text { Superior a Distancia }\end{array}$ & $1981-1988$ & $5 / 24$ & 85 \\
\hline $\begin{array}{l}\text { Revista Iberoamericana de Educación } \\
\text { Superior a Distancia }\end{array}$ & $\mathbf{1 9 8 8}$ - 1996 & $8 / 23$ & 174 \\
\hline $\begin{array}{l}\text { RIED. Revista Iberoamericana de } \\
\text { Educación a Distancia }\end{array}$ & 1998 - 2018 & $21 / 42$ & 440 \\
\hline Total & $\mathbf{3 7}$ años & $\mathbf{3 4 / 8 7}$ & $\mathbf{6 9 9}$ \\
\hline
\end{tabular}

Dado que el referente en este trabajo es la celebración del 30 aniversario de la RIED, contemplemos sólo las dos revistas científicas, excluyendo el Boletín Informativo. En este caso, en los 30 años de RIED/RIESAD, se han publicado 29 volúmenes, 65 números y un total de 614 artículos.

Ese recorrido no supone otra cosa que lo que ha sido la evolución o tránsito desde la universidad convencional, "analógica", a la universidad digital. Precisamente por eso, porque estamos apostando por esa universidad digital es por lo que este número actual de la RIED recoge un monográfico coordinado por Manuel Area y dedicado a esa temática.

Actualmente se está trabajando en un estudio bibliométrico de estos 30 años de publicación científica de la AIESAD que esperemos vea la luz en el próximo número de la RIED. Igualmente, hemos incorporado todos los artículos de la primera etapa de la revista (RIESAD) 1988-1996 en la plataforma OJS de la RIED, su sitio web 
oficial, con el fin de que se pueda tener acceso a la publicación íntegra de estos 30 años de historia.

Nos sentimos felices y orgullosos de que, junto a tantos profesores einvestigadores comprometidos de forma desinteresada en el mantenimiento de esta publicación, cuyos nombres más destacados hemos tratado de recoger en este artículo, y junto a los autores, miembros de comités científico y de redacción, revisores internacionales $\mathrm{y}$, en fin, lectores de todas las zonas del mundo, podamos estar celebrando nada menos que 30 años de una publicación científica. Ello ha sido posible gracias a todos los implicados de una u otra forma en las tres publicaciones sucesivas que han sido motivo de análisis evolutivo en este trabajo y que la AIESAD con gran previsión del futuro supo patrocinar. Pero esta asociación internacional debería incrementar sus esfuerzos para potenciar a esta revista científica. Las publicaciones periódicas científicas de hoy precisan de muchos recursos con el fin de salvaguardar la calidad, la transparencia y la independencia. Esperamos que la AIESAD sepa responder a este reto de forma decidida.

Continuaremos en el empeño y, en su momento, a los que puedan sucedernos en esta apasionante tarea de gestionar la RIED, pedirles que no decaigan en el ánimo, fundamentalmente, por el bien de la innovación en la educación, sea ésta presencial o a distancia.

\section{REFERENCIAS BIBLIOGRÁFICAS}

García Aretio, L., y Ruiz Corbella, M. (2011). Del boletín informativo de la Asociación Iberoamericana de Educación Superior a Distancia a la Revista Iberoamericana de Educación a Distancia (RIED): la aportación de AIESAD a la investigación sobre educación a distancia en América Latina. En $X X X$ Aniversario de la AIESAD: la Asociación Iberoamericana de Educación Superior a Distancia (AIESAD), pionera en la divulgación científica sobre educación a distancia en Latinoamérica. Madrid: UNED.

García Pérez, M. (2011). La Revista Iberoamericana de Educación Superior a Distancia (RIESAD). En $X X X$ Aniversario de la AIESAD: la Asociación Iberoamericana de Educación Superior a Distancia (AIESAD), pionera en la divulgación científica sobre educación a distancia en Latinoamérica. Madrid: UNED.
Miranda, A., y Yee, M. (2014). Educación a Distancia en Iberoamérica: XXX aniversario de la AIESAD. RIED. Revista Iberoamericana de Educación a Distancia, 13(2), 13-36. doi: https://doi. org/10.5944/ried.13.2.1345.5

Rivas, R. (2011). RIED. Revista Iberoamericana de Educación a Distancia. En XXX Aniversario de la AIESAD: la Asociación Iberoamericana de Educación Superior a Distancia (AIESAD), pionera en la divulgación científica sobre educación a distancia en Latinoamérica. Madrid: UNED.

Varela, S. (2011). Boletín Informativo de la Asociación Iberoamericana de Educación Superior a Distancia: primera publicación de AIESAD. En XXX Aniversario de la AIESAD: la Asociación Iberoamericana de Educación Superior a Distancia (AIESAD), pionera en la divulgación científica sobre educación a distancia en Latinoamérica. Madrid: UNED. 


\section{Como citar este artículo}

García Aretio, L. (2018). RIED. Una historia de 30 años de publicación científica. RIED. Revista Iberoamericana de Educación a Distancia, 21(2), pp. 09-21. doi: http://dx.doi.org/10.5944/ried.21.2.21273 